\title{
ATPG With Efficient Testability Measures and Partial Fault Simulation
}

\author{
Kamal Kumar Jain* James Jacob ${ }^{\dagger} \quad$ Srinivas $M \mathrm{~K}^{\dagger}$ \\ Indian Institute of Science, Bangalore 5 GO 012, India
}

\begin{abstract}
In this paper we propose an improved version of the test generation algorithm PODEM (Path Oriented DEcision Making) incorporating a different technique for back tracing and forward implication. We also propose a partial fault simulatos. which is integrated into the improved PODEM algorithm. The performance of this test generation package (when partial fault simulator is employed) is compared to that of a concurrent fault simulator using deterministically generated test patterns. It is shown that the runtime performance of our algorithm compares favisulally with that of the concurrent fault simulator and is less memory intensive. We also present effective heuristics to determine some of the redundant faults and to derive the test vectors for some PI faults, by the use of implication relations. Experimental results on all tlie 10 ISCAS benchmark circuits [7jdemonstrate that our algorithm is faster and more eflicient t $t_{1 \mathrm{a}}$ the PODEM algorithm for tlicse circuits.
\end{abstract}

\section{Introduction}

Many test generation algorithms have been proposed over the years. Recent algorithms such as PODEM [2], FAN [3], SOCRATES [4]etc. have been sucessful in generating test patterns with reasonableefficiency. We assume that the reader is familiar with tlie PODEM algorithm and we shall use some terminologies such as $D, \bar{D}$, PI, PO, backtracing, backtracking, forward implication, etc. without definitions. In this paper, we shall consider multi-input multi-output combinational circuits composed of AND, OR, NAND, NOR, NOT, BUFFER, XOR, and XNOR gates. The type of fault model assumed here is the standard single stuck fault, i.e., all faults can be modeled by lines which are stuck at logical $0(\mathrm{~s}-\mathrm{a}-0)$ or stuck at logical $1(\mathrm{~s}-\mathrm{a}-1)$. Only one line is assumed to be faulty at any given instant.

* Computer Science and Automation

Piscetical Commmication Engs.

tComputer Aided Design Lab

\section{Improved PODEM}

\subsection{Modification 1}

In the original paper on the PODEM algorithm XOR and XNOR gates were not considered anywhere in the flowcharts, although mentioned in an example given in tlie paper. In this algorithm we have included XOR and XNOR gates also.

\subsection{Modification 2}

If in tlie imply process, the faulty line is set to a logic level which is the same as stuck at, level, then backtracking should be done immediatcly, which will lead to faster excculion. This point, which is missing in the flowchart of tlie original PODEM description [2], lias been taken care of in our implimentation.

\subsection{Modification 3}

PODEM algorithm uses heuristics to guide the backtracing and implication processes that rely on estimates of the "ease of controlling" internal lines .of a circuit to certain logic values, and on estimates of tlie "ease of observing" values on internal lines at primary outputs(POs) of the circuit. Since test for a stuck at fault on a line requires both controlling tlie line to a certain logic value and observing this value at a PO, controllability and observability are usally combined under the more geueral notion of testability. PODEM algorithm seems to lack in the careful consideration of the point of controllability. An example of such a case is given in figure 1 .

Consider the logic circuit of figure 1 . Let 0 be the objective logic value at the output of tlie gate $M$. In this example PODEM will choose tlie upper path in the process of backtracing [2]. But actually, the lower path is mucli more easier to control from tlie PIs, because to set a 0 at the output of the gate $\mathrm{K}$ either $\left(I_{1}, I_{2}, I_{3}, I_{1}\right)$ or $\left(I_{3}, I_{4}, I_{5}, I_{6}\right)$ should be set to $(1,1,1,1)$, while a 0 at the output of gate $\mathrm{I}_{3}$ can be obtained by setting only either $I_{9}$ or $I_{10}$ to 0 . This problem arises due to the 


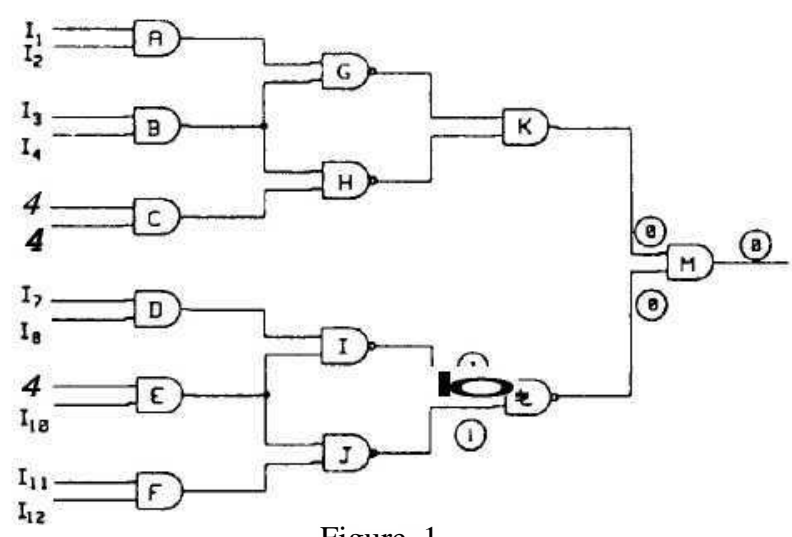

Figure 1

fact that PODEM looks only at one level lower (at the inputs only) in choosing a path, which may be a non-optimal decision, as shown in the last example.

So, it will be better if all the paths from the objective line to tlie PIs can be considered arid tlie appropriate path chosen.

\subsubsection{Algorithms For Testability Measures}

There are a number of algorithms available for determining the controllability and observability for every line of the circuit. Some of the algorithms are CAMELOT, COMET, TMEAS, SCOAP, and VICTOR [1]. Tlie most popular among them is the "SCOAP" algorithm [5]. In this paper, Testability Measures have been used in order to speed up the test generation algorithm. Controllabilities and observabilities are calculated before the start of tlie test generation procedure. Controllability measures have been used in "easiest" and "hardest" functions [2] of PODEM and observability measures have been used in order to determine the X - path after the imply process [2]. We have introduced tlie SCOAP controllability measures to guide the backtracing process of the test generation algorithm. Since SCOAP is fairly well known; we do not discuss the details of how to obtain tlie controllability measures.

In the example of figure 1, 0-controllabilities of nodes $\mathrm{K}$ and $\mathrm{L}$ are calculated as $\mathrm{S}$ and 7 respectively, so now our algorithm will choose tlie lower path.

It was found experimentally that introduction of observability measures did not yield any definite advantage and hence it was abandoned. Ilowever a different heuristic was developed to guide the forward implication process and is explained below.

\subsection{Modification 4}

A preprocessing was done before tlie begining of tlie test generation procedure, which stores the information at each line about the POs which are likely to be affected by this line. So, when an X-path from a line is to be found, first, tlie logic level of POs which arc affected by this line are checked. If no PO is at X then this implies that there exists no X-path from that line to any PO of the circuit, and immediately bicktracking is performed, wliicli will lead to considerable savings in computation time. The rosults obtiained with this modification (preprocessing for $\mathrm{X}$-path) were very effective. So, this modification was retained and the modifications using obscrvnbility monsures nere discarded.

Tlie results obtained after combining preprocessing for the X-path and modifications for the controllabilty measures are shown in table 1 and table $2[9]$. The test vectors were minimised by a simple strategy and their number is also reported in table 1 .

\section{Fault Simulator}

Tlie fault coverage of a set of test vectors is measured through fault simulation. A combination of test generation and fault simulation is effective in speeding up the test generation process. Hence a partial fault simulator which will run along with the test generation algorithm was developed and integrated into our algorithm. The basic strategy is to employ a partial fault-simulation after each test vector (for a fault) is generated, so as to eliminate the covered fults from tlie fault list. Usually faults detected by a given test are dropped from the fault list. This is known as fault dropping. Note that a test vector can be a test for more than one fault.

Parallel, Concurrent, and Deductive Fault simulators[1] require excessive memory and CPU time as circuit size increases. The partial siinulator developed and implemented by us is iiot based on any of tlie above three. This partial fault simulation draws upon and extends the ideas of TEST DETECT [S] developed by tlie inventors of tlie D - algorithm.

An extended version of the partial fault simulator was also developed in course of our work. A comparison was made between this one and tlie earlier partial fault simulator. It was obsrrvetl tlint al though the partial one had to generate more number of test vectors than the extended one, it was much more efficient in terms of CPU time than the latter. However, the extended version is also supported by our package as a user option and can be used if minimality of test set is crucial. 
Table 1:Times and Test set size on SUN 3/60

\begin{tabular}{|c|c|c|c|c|c|c|c|c|c|c|c|}
\hline \multirow{3}{*}{ Circuit } & \multirow{3}{*}{$\begin{array}{l}\text { Total } \\
\text { Faults }\end{array}$} & \multirow{3}{*}{ Gates } & \multirow{3}{*}{ PI } & \multirow{3}{*}{ PO } & \multirow{2}{*}{\multicolumn{4}{|c|}{$\frac{\text { CPU time in Sec. }}{\text { Methods }}$}} & \multirow{2}{*}{\multicolumn{3}{|c|}{$\frac{\text { Minimal Test Size }}{\text { Methods }}$}} \\
\hline & & & & & & & & & & & \\
\hline & & & & & $\mathrm{A}$ & B & C & D & $B$ & C & $\mathrm{D}$ \\
\hline 74181 & 182 & 86 & 14 & $\mathrm{~S}$ & 4 & 4 & 3 & 5.7 & 37 & 31 & 31 \\
\hline C432 & 524 & 160 & 32 & 7 & 98 & 92 & 52 & 67 & 122 & 109 & 99 \\
\hline C499 & 758 & 202 & 41 & 36 & 197 & 183 & 74 & 186 & 358 & 149 & 126 \\
\hline $\mathrm{C} 880$ & 942 & 383 & 60 & 26 & 76 & 62 & 43 & 82 & 101 & 90 & 90 \\
\hline C1355 & 1574 & 546 & 41 & 32 & 662 & 590 & 274 & 1785 & 315 & 270 & 176 \\
\hline C1908 & 1879 & 880 & 33 & 25 & 640 & 620 & 374 & 1854 & 435 & 359 & 271 \\
\hline $\mathrm{C} 2670$ & 2747 & 1193 & 233 & 140 & 1091 & 1020 & 886 & 3238 & 206 & 184 & 146 \\
\hline C3540 & 3428 & 1669 & 50 & 22 & 2105 & 1668 & 1276 & 4796 & 439 & 392 & 307 \\
\hline C531S & 5350 & 2307 & 178 & 123 & 3068 & 2354 & 1425 & 4742 & 400 & 345 & 261 \\
\hline C62S8 & 7744 & 2406 & 32 & 32 & 8726 & 8081 & 2515 & $>15 \mathrm{Hrs}$ & 1661 & 1074 & - \\
\hline C7552 & 7550 & 3512 & 207 & 108 & 11148 & 8277 & 4985 & 20374 & 724 & 558 & 353 \\
\hline
\end{tabular}

A: Basic PODEM algorithm

Timeout value per fault $=2 \mathrm{sec}$

$\mathrm{B}$ : New PODEM = PODEM + controlnbility + preprocessing

C: New PODEM + partial fault simulator

D: New PODEM + extended fault simulator

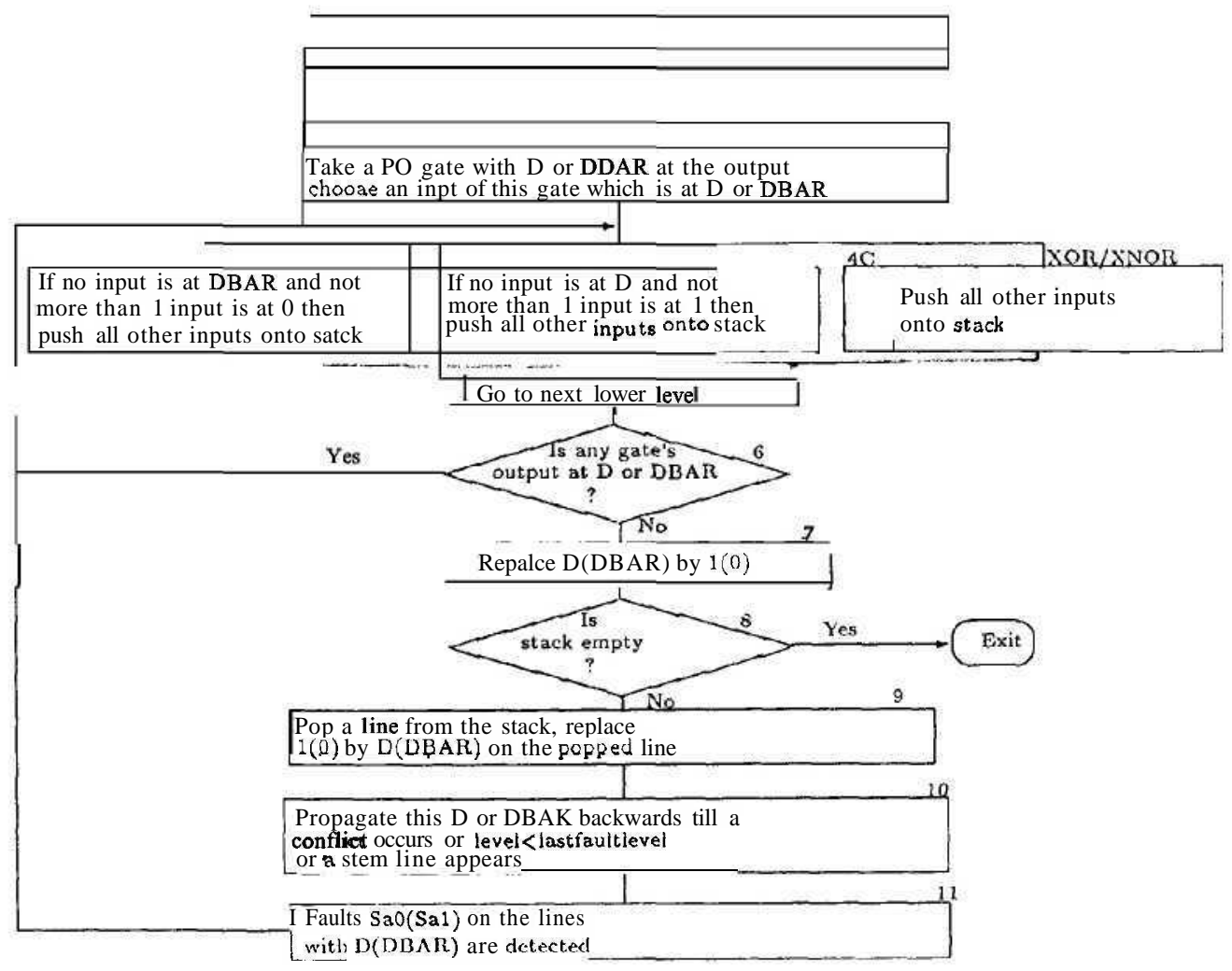

Figure 2: Flow chart of Partial Fault Sin-iulator 


\subsection{Partial Fault Simulator}

Tlie flowchart of the algorithm, developed for tlie partial fault simulator is shown in figure 2 . Tlie explanation of every box is given below:

Box 1: A test vector is chosen, wliicli isjust generated by the PODEM algorithm.

Box 2: The test vector will be able to detect all tlie $\mathrm{saO}$ (sal) faults on the lines, along asensitized path to a PO and having a $D(\bar{D})$ logic value. This is easily understood from the basic concept of path sensitization. Box 3: Now a PO gate is taken which is having a $D$ or $\bar{D}$ on its output. Beacuse the output is at $D$ or $\bar{D}$ at least one input will be at $\mathrm{D}$ or $\mathrm{D}$. Choose such an input.

Box 4: Depending upon the function of the gate, other inputs of tlie gate are pushed onto the stack for the purpose of backward implication of $D$ or $\bar{D}$. But, for example, in tlie case of AND/NAND gate (box 4A), if any input is at $\bar{D}$ then any other input, cannot be pushed onto tlie stack, because $\bar{D}$ is being replaced by a.O in tlie backward implication and in that case tlie output value of the gate will not bo at $D$ or $\bar{D}$.

Boxes 5 and G: Tlie loop of the boxes 5,6, and 4 is esecuted till all the posible lines for which backward implication can be performed are over.

Boxes 7,8,9, and 10: A line is popped and a 1(0) is replaced by $D(\bar{D})$ on the popped line and this $D$ or $D$ is propagated backward till a conflict occurs or the current circuit level becomes less than tlie level of the faulty line for which test vector was generated or a fanout stein line appears. Stopping at fanout stem line is one of tlie reasons, why this fault simulation is partial. The backward implication is stopped at the level less than the faulty line level because of tlie definite order of the faults in tlie fault list.

Tlie order of the faults in tlie fault list is giveii below:

1. All the faults of the PIs.

2. The output faults of the next higher level.

3. The input faults of this level.

4. The output faults of the nest higher level and so on.

So, when current level has come down to less tlian tlie faulty line level for which tlie test vector was generated, then there is no point in going further back because tlie faults of the nest lower level would have been considered earlier. This saves a lot of time.

Box 11: this box is similar to box 2 .

Example: Tlie values at each line of the circuit after generating the test vector $(0 x 10)$ for the fault, $1 \mathrm{~s}-\mathrm{a}-1$ by PODEM is shown in figure 3 . From box 2 of the flowchart it is clear that $15 \mathrm{~s}-\mathrm{a}-0,14 \mathrm{~s}-\mathrm{a}-0, \mathrm{G} \mathrm{s}-\mathrm{a}-1$, and $1 \mathrm{~s}-\mathrm{a}-1$ are testable by this test vector. According to

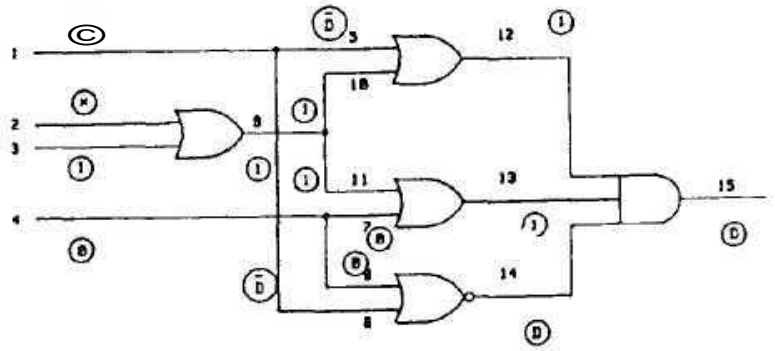

Figure 3:

box $4 \mathrm{~A}$, push line 12 and 13 onto the stack. Line 14 is at $D$; so according to box $4 \mathrm{~B}$ push line $S$ on to the stack. Now replace all $D(D)$ by $1(0)$ in the circuit. A line is popped from the stack. This is line number 8 and having a logic: value of 0 . Repplace llis 0 by a I). Line 4 is a fanout stem line so according to box 10 this $D$ can not be propagated to line $4.8 \mathrm{~s}-\mathrm{a}-1$ is detected by this test vector (box 11). Similar procedures result. in delecting $12 \mathrm{~s}-\mathrm{a}-0,10 \mathrm{~S}-\mathrm{a}-0,13 \mathrm{~s}-\mathrm{a}-0$, aiid $11 \mathrm{~s}-\mathrm{a}-0$. Thus tlie test vector $(0 \times 10)$ could detect 9 faults.

\subsection{Other Features Of The Package}

\subsubsection{Implication For PI Faults}

If for a PI both stuck-at-0 and stuck-at-1 faults are included in tlie fault list, then tlie test vector lor only one type of fault needs to be generated. Test vector for tlie other type of fault (opposite type) can be determined just by complementing tlie bit (PI) for wliicli the fault, is being considered. For example, in the last example circuit, tlie test vector $(1 \times 10)$ for line $1 \mathrm{~s}-\mathrm{a}-$ 0 can be generated just. by implication, if test vector (0x10) for $1 \mathrm{~s}-\mathrm{a}-1$ has already been generated.

\subsubsection{Implications For Untestable Faults}

Three observations:

1. If a $\mathrm{saO}$ (sal) fault on a PI is untestable then sal(sa0) fault on that PI is also untestable.

2. If a fault / is untestable, all faults in its equivalence class are also uiitcstable.

3. If a dominating fault is untestiable, all faults dominated by this untestable fault are also untestable.

The truth of these observations is obvious and hence no formal proof is given.

Example: If stuck-at-0 fault, at the input of an AND 


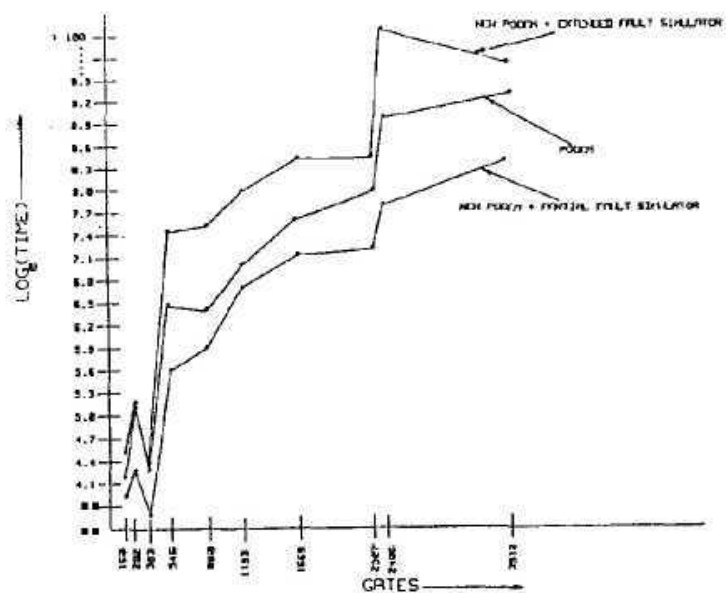

Figure 4: Performance of different versions

gate is found untestable then the stuck-at- 0 faults on all other inputs and the output of that gate will be untestable. These faults are declared untestable by the property of fault equivalence.

This property can be propagated further forward and backward until a fanout stem line appears. For example, in tlie last example circuit, faults $10 \mathrm{~s}-\mathbf{a}-1$ and $5 \mathrm{~s}-\mathrm{a}-1$ can be declared untestable by the implication of $12 \mathrm{~s}-\mathrm{a}-1$ being untestable.

This particular feature of the pacltage is very important since in general tlie untestable faults are hard to prove redundant and PODEM will take a lot of time to deal with these faults. So, without going into the test generation program, many of these type of faults are declared untestable just by implication.

\subsubsection{Extended Version Of Partial Fault Sim- ulation}

Tlie difference in the partial fault simulation and tlie extended partial fault simulation appears only in the box 10 of the flowchart shown in figure 2 . In partial fault simulation the backward implication of $\mathrm{D}$ or $\bar{D}$ stops at a fanout stem line, while in tlie extended partial fault simulation, after setting the fanout stem line to $D$ or $\bar{D}$ (as may be the case), a forward implication is performed and if the logic value of the PO which was coiisiderd in tlie begining of the simulation remains unchanged at $D$ or $\bar{D}$ then tlie backward implication is continued from the fanout stem. The forward implication consumes a large amount of time making tlie extended partial fault simulation inefficient. The results for both the versions are shown in table 1 and table 2 .

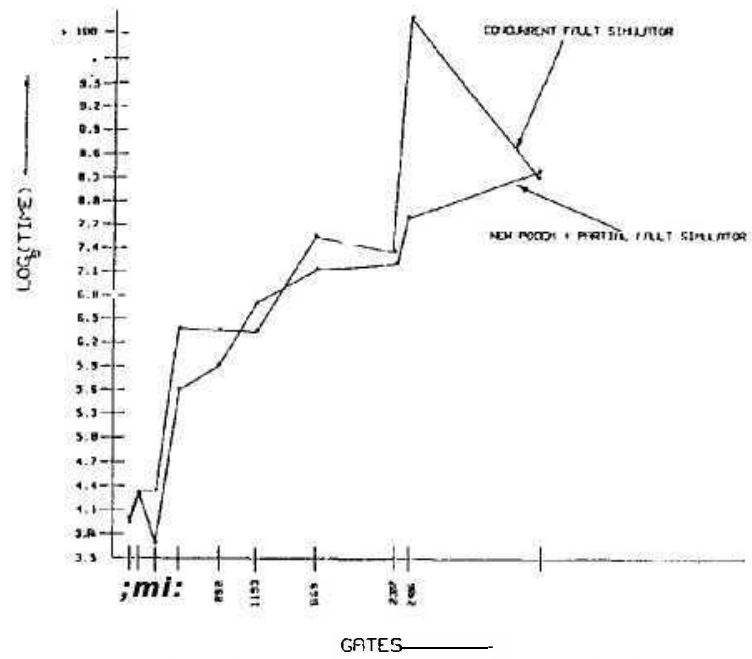

Figure 5: Time comparison with CFS

\section{Results}

Tlie improved PODEM algorithm along with the integrated fault simulator was coded in $\mathrm{C}$ and implemented on a SUN 3/60 workstation. It, consists of about 2000 lines of code. Tlie efficiency of our shralegy of integrating a partial fault simulator into an improved version of PODEM algorithm is demonstrated by comparing its performance to that of a Concurrent fault simulator for all tlie ISCAS benchmark circuits. Fault simulation is generally known to be less expensive compared to computation intensive test generation process, as we do not have baclitraclting in fault simulation. Tlie test vectors derived by PODEM (employing Partial Fault Simulation) were given to CFS, a concurrent fault simulator available in the CAD Lab. at IISc. The simulator evaluated the coverage of the deterministic test vectors generated by PODEM for all the 10ISCAS benchmark circuits. Contrary to our expectations it was found that in many cases the time taken by CFS to evaluate the coverage of tlie test. vectors was greater than tlie time taken by our package to generate the same vectors. The results are shown in table 3. CFS is memory intensive and hence it ran out of virtual meniory for C6288 on a SUN 3/60 workstation with $4 \mathrm{MB}$ main memory, while our PODERI implimentation could handle all tlie benchmark circuits. Tlie results clearly demonstrate that a combination of test generation and fault simulation can compare favourably in performance to that of a stand alone fault simulator, even though deterministic test generation is more compute intensive as it employs backtracking. The concurrent fault simulation naturally reported a higher fault coverage than that determined 
Table 2: Fault coverage with different versions on SUN 3/00

\begin{tabular}{|c|c|c|c|c|c|c|c|c|c|c|c|c|c|}
\hline \multirow{3}{*}{ Circuit } & \multirow{3}{*}{$\begin{array}{l}\text { Total } \\
\text { Faults }\end{array}$} & \multicolumn{4}{|c|}{ Faults Aborted } & \multirow{2}{*}{\multicolumn{4}{|c|}{ Faults Redundant }} & \multicolumn{4}{|c|}{ Litult Coverage (//) } \\
\hline & & \multicolumn{4}{|c|}{ Methods } & & & & Methorls & \multicolumn{4}{|c|}{ Methods } \\
\hline & & A & $\mathrm{B}$ & $\mathrm{C}$ & $\mathrm{D}$ & A & $\mathrm{B}$ & $\mathrm{C}$ & $\mathrm{D}$ & A & $\mathrm{B}$ & $\mathrm{c}$ & $\mathrm{D}$ \\
\hline 74181 & 182 & 0 & 0 & 0 & 0 & 1 & 1 & 1 & 1 & 99.50 & 99.50 & 99.50 & 99.50 \\
\hline C432 & 524 & 26 & 26 & 13 & 13 & 0 & 0 & 0 & 0 & 95.04 & 95.04 & 97.52 & 57.52 \\
\hline C499 & 758 & $2 \mathrm{G}$ & $2 \mathrm{G}$ & $1 \mathrm{G}$ & 20 & 0 & 0 & 0 & 0 & 96.57 & 06.57 & 97.9 & 97.34 \\
\hline $\mathrm{C} 880$ & 942 & 0 & 0 & 0 & 0 & 0 & 0 & 0 & 0 & 100.00 & 100.00 & 100.00 & 100.00 \\
\hline C1355 & 1574 & 8 & 16 & 8 & 8 & 0 & 0 & 0 & 0 & 99.49 & 98.98 & 99.49 & 96.49 \\
\hline C1908 & 1879 & 28 & 57 & 41 & 37 & 4 & 5 & 5 & 5 & 98.30 & 93.60 & 97.60 & 97.80 \\
\hline $\mathrm{C} 2 \mathrm{~S} 70$ & 2747 & 118 & 147 & 138 & 103 & 27 & 29 & 29 & 29 & 94.70 & 93.60 & 93.90 & 95.20 \\
\hline C3540 & 3428 & 384 & 301 & 229 & 181 & 15 & 31 & 31 & 31 & 88.4 & 90.3 & 92.40 & 93.80 \\
\hline C5315 & 5350 & 200 & 127 & 98 & 97 & 36 & 36 & 38 & 36 & 95.60 & 97.00 & 97.50 & 97.50 \\
\hline C6288 & 7744 & 111 & 111 & 12 & & 32 & 32 & 32 & & 98.20 & 98.80 & 09.10 & - \\
\hline C75S2 & 7550 & 2475 & 1162 & 949 & 771 & 48 & 52 & 52 & 52 & 66.60 & 83.00 & 86.70 & $89 . \mathrm{JO}$ \\
\hline
\end{tabular}

A: Basic PODEM algorithm

C: New PODEM + partial fault simulator
D: New PODEM = PODEM + controlability + preprocessing

D: New PODEM + extended fault simulator Timeout value per lault $=2 \mathrm{~s} \times \mathrm{c}$ by PODEM and this is easily explained by the fact that the simulator we have integrated into PODEM is a partial one.

\section{Conclusions}

The results indicate that our algorithm is better than the basic PODEM algorithm. 'lestabillity IIIwsures are very effective. A partial fault simualtor is more effective than a complete fault simulator. Some morc modifications can make this algorithm more efficient. Some of these modifications are multiple path backtracing[3], unique sensilization [3], identification techniques for the redundant faults [6] and a better fault simulator.

\section{Acknowledgement}

The authors would like to tha.nk Prof. Sharad C. Seth of University of Nebraska, Lincoln for providing a Pascal implementation of basic PODEM algorithm and a concurrent fault simulation package called CFS.

\section{References}

[i] V.D. A garwal and Shavad C. Seth, "Test Generation for VISIChips." Tutorial, IEEL Computor Society Pless, Computer Society Ordor Number 7S6, 1088.

[2] P. Gocl, "An Implicit Eummeralion Algorithm Io Gence ate Tests For Combinational Logic Circuits," lWEL Trans, Computers, pp. 215-222, Marcli,1981.

[3] H. Fujivara and T. Shimono, "On The Acceleration of Test Generation Algoritum," IELE Trans. Compulers, pp 1137-1155,Dec. 1983
Table 3: CPU time comparison on SUN 3/60

\begin{tabular}{|c|c|c|}
\hline Circuit & $\begin{array}{c}\text { New PODEM + } \\
\text { Partial Fault } \\
\text { Siluulator }\end{array}$ & $\begin{array}{l}\text { Concurrent Fault } \\
\text { Simulator }\end{array}$ \\
\hline C4132 & $52 \mathrm{Scc}$ & $5 \bar{t} \mathrm{Sec}$. \\
\hline C490) & 74 Sec. & 75 Sec. \\
\hline Q880 & 43 Sec. & t. ScC. \\
\hline $\mathrm{C} 1355$ & 274 Sec. & $5 \mathrm{so}$ Sec. \\
\hline C1008 & $374 \mathrm{Scc}$ & $570 \mathrm{Src}$. \\
\hline 0.26711 & Sosis sica: & [Niil Sen \\
\hline $0.35 \cdot 10$ & I276 Sca: & 1817 Sec: \\
\hline C5315 & $1425 \mathrm{Sec}$. & $1566 \mathrm{Sec}$. \\
\hline C6288 & 2515 Sec. & $\begin{array}{l}\text { Not. Completed } \\
(\text { lime }>20 \text { llis.) }\end{array}$ \\
\hline C75S2 & AgSE Sec. & $31219 \sin$. \\
\hline
\end{tabular}

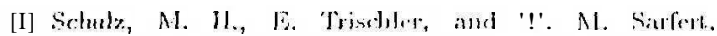
"SOCIRATIS: A Jlighely Ellicient Automatis Piat l'atlmon Generation Systems," 1EEE'Trans. on CAD, pp. 126-137. Jan.198s.

[5] L. H. Goldstein and E. L. Thigpen,"SCOAP: Sandia Controllability/Olsscrability Analysis Progran," Proc. J Tih DCS. Auto. Cont. Minneapolis, MN, pp, 150-106, Jume: 1980.

[G] M. 11. Sclulz and li. Auth, "Advancesl Automatic 'Irst. Pattern Generation and Redundancy Identification Techniques," IEEE FTCS 1988.

[7] F. Biglez and H. Fujiwara, "A Neutral Netlist of 10 Combinationnl Benchmark Circuits and a Target Translator in Fortran," Proc. IEEE Int. Symposium on Circuits and Systems; Spucial Session on N'PG aurl Fault Simulation. Jume 1985.

[8] J.P. Rolh, "Programmed Mrorillums to Compule Tests to Detect and Distinguish betwern lizilures in Logic Circuits," IFEF Trans. Computer, vol. EC-16. 1w.5. Oct. 19G7, pp. $566-580$

[9] Kamal Kumar Jain, "Developuent of an ellicicnt software package for Test Generation of Combinational Circuits," M.E Thesis. Dept, of Electrical Communimation Buginerring, Indian Jnstitute of Science. Bangalote, June 1990 